\title{
Peroral endoscopic pyloromyotomy accelerates gastric emptying in healthy pigs: proof of concept
}

Authors

Institutions
Sophie Geyl', Romain Legros ${ }^{1}$, Aurélie Charissou² ${ }^{2}$, Laura Mesturoux ${ }^{2}$, Claude-Yves Couquet ${ }^{3}$, Paul Carrier ${ }^{1}$, Anaïs Brayette ${ }^{1}$, Zora El-Ouafi ${ }^{1}$, Veronique Loustaud-Ratti ${ }^{1}$, Denis Sautereau ${ }^{1}$, Jacques Monteil ${ }^{4}$, Jérémie Jacques ${ }^{1}$

${ }^{1}$ service d'hépato-gastro-entérologie, CHU Dupuytren, Limoges, France

${ }^{2}$ service d'anatomopathologie, CHU Dupuytren, Limoges, France

${ }^{3}$ laboratoire de recherche et d'analyses de la Haute-Vienne, Limoges, France

${ }^{4}$ service de médecine nucléaire, CHU Dupuytren, Limoges, France submitted

23. January 2016

accepted after revision

2. May 2016

\section{Bibliography}

Dol http://dx.doi.org/

10.1055/s-0042-108192

Published online: 29.6.2016

Endoscopy International Open

2016; 04: E796-E799

(c) Georg Thieme Verlag KG

Stuttgart · New York

E-ISSN 2196-9736

\section{Corresponding author}

\section{Jérémie Jacques, MD}

service d'hépato-gastroentérologie

CHU Dupuytren

2 avenue Martin Luther-King 87042 Limoges

France

Fax: +33-5-55058733

jeremiejacques@gmail.com
Introduction: Gastroparesis, or delayed gastric emptying, can be diagnosed with gastric emptying scintigraphy. Manometric studies of patients with gastroparesis show increased pyloric tone (pylorospasm). Among the recent endoscopic therapies for pylorospasm is peroral endoscopic pylorotomy (POP). In this study, we explored the effect of POP on gastric emptying in healthy pigs. Material and methods: Four mini-pigs underwent POP following general anaesthesia. The mucosal entrance was situated $5 \mathrm{~cm}$ above the pylorus. POP was performed through a submucosal tunnel dissection. The duration of gastric emptying was assessed by scintigraphy before and after the procedure. The pigs were then euthanised for necropsy and pathologic assessment of the pylorus. Results: The mean duration of the procedure was $55( \pm 4 \mathrm{SD}) \mathrm{min}$. All surgeries were performed in

\section{Introduction}

$\nabla$

Gastroparesis is characterised by an association with upper gastrointestinal symptoms and objective delayed gastric emptying in the absence of mechanical obstruction [1]. It occurs in $1.8 \%$ of the population [2], with a higher prevalence in women. Symptoms include early satiety, postprandial fullness, nausea, vomiting, and upper abdominal pain. These symptoms are essentially induced by eating and often lead to an altered quality of life [3] or nutritional deficiencies. The gold standard for the diagnosis of gastroparesis is gastric emptying scintigraphy, performed after the consumption of a standardised radiolabelled meal [4].

Gastroparesis is most often idiopathic, but it can also develop in the setting of diabetes or postoperatively due to vagal damage during surgery [5]. Most treatments of gastroparesis, such as pro- their entirety with $100 \%$ feasibility. There were no cases of bleeding. The one case of perforation had no clinical significance. The duration of gastric emptying was 2.22-fold shorter after POP compared with before POP $(T 1 / 2$ post-POP $=84.5$ $[ \pm 35.7 \mathrm{SD}] \mathrm{min}$ vs. $T^{1} / 2$ pre-POP $=188.4[ \pm 87.3 \mathrm{SD}]$ min; $P=0.029$ ). In agreement with the endoscopic observations, sectioning of the pyloric muscle in each pig was histologically complete.

Conclusion: The efficacy of the procedure provides indirect proof of the involvement of the pyloric ring in delayed gastric emptying and suggests new therapies for patients with gastroparesis. Our protocol combining gastric emptying scintigraphy and POP validated the use of anaesthetised mini-pigs as a learning and training model for POP or other endoscopic/surgical procedures related to gastric emptying.

kinetics, target the symptoms and thus are frequently ineffective. Surgical therapies include gastric electric stimulation, but evidence for the efficacy of this approach is lacking. Recent manometric studies have shown that pyloric pressure, including phasic and tonic contractions, is elevated in patients with gastroparesis [6,7]. This phenomenon, termed pylorospasm, has resulted in new therapeutic approaches in patients who develop gastroparesis after surgical pyloroplasty [8]. Inspired by the success of peroral endoscopic myotomy (POEM) [9] in patients with achalasia, the use of peroral pyloromyotomy (POP) in a patient with refractory gastroparesis was first described in 2013 [10]. However, POP is difficult to perform, especially by physicians without experience in POEM. Here, we describe a porcine model suitable for training physicians in POP. We also explore the effect of POP on gastric emptying in healthy pigs. 

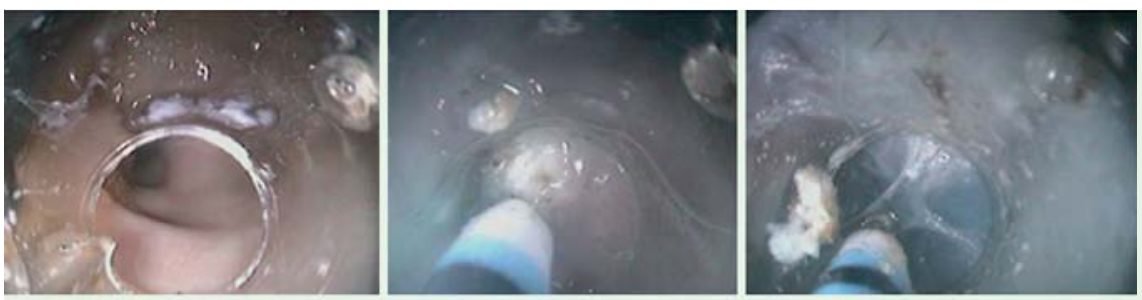

Fig. 1 Peroral pyloromyotomy. a, pyloric ring;

b, submucosa of the bulb; c, mucosa of the bulb.
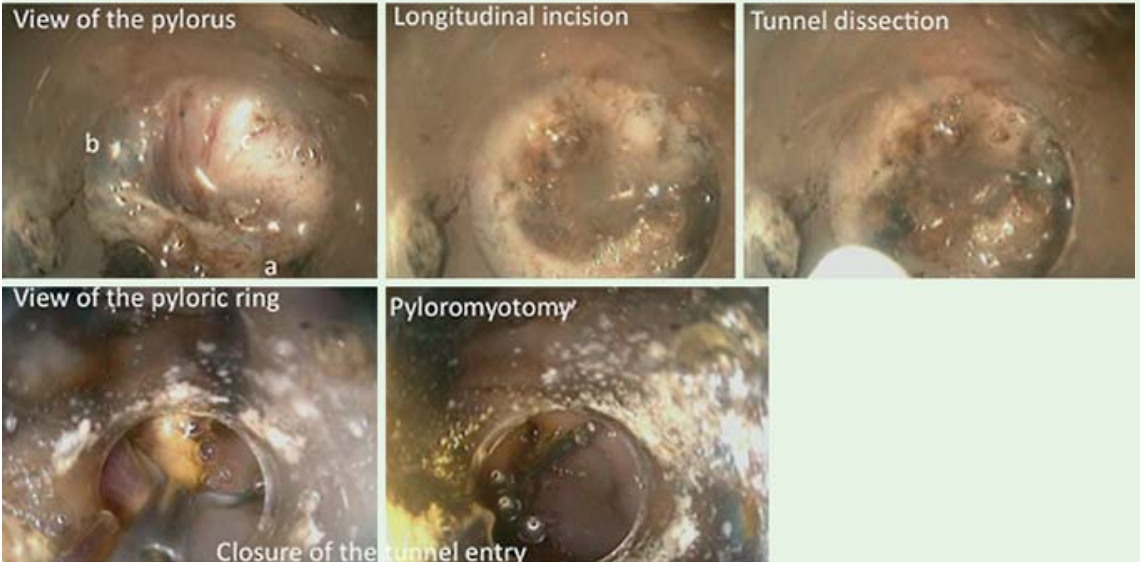

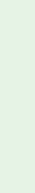


Table 1 Results for peroral endoscopic pylorotomy and gastric emptying.

\begin{tabular}{|c|c|c|c|c|c|}
\hline & Pig 1 & Pig 2 & Pig 3 & Pig 4 & Mean \\
\hline Procedure duration, min & 55 & 50 & 55 & 60 & $55( \pm 4.08 \mathrm{SD})$ \\
\hline \multicolumn{6}{|l|}{ Complications, n } \\
\hline Perforation & 1 & 0 & 0 & 0 & $25 \%$ \\
\hline Bleeding & 0 & 0 & 0 & 0 & $0 \%$ \\
\hline Anticipated sacrifice & 0 & 0 & 0 & 0 & $0 \%$ \\
\hline \multicolumn{6}{|l|}{$T 1 / 2$ gastric emptying, $\min$} \\
\hline Before POP & 301.89 & 89 & 180 & 183 & $188.47( \pm 87.3 \mathrm{SD})$ \\
\hline After POP & 125 & 38 & 85 & 90 & $\begin{array}{l}84.5( \pm 35.74 \text { SD }) \\
P=0.03(C I 95 \%: 20.15-187.8)\end{array}$ \\
\hline Technical difficulty & Loop +++ & Loop + & Loop ++ & Loop ++ & \\
\hline Feasibility, $\mathrm{n}$ & 1 & 1 & 1 & 1 & $100 \%$ \\
\hline \multicolumn{6}{|l|}{ Pathological analysis } \\
\hline \% section of pyloric muscle, $\%$ & $>90$ & $>90$ & $>90$ & $>90$ & $>90$ \\
\hline
\end{tabular}

POP, peroral endoscopic pylorotomy.

action amoxicillin $(25 \mathrm{mg} / \mathrm{kg})$ administered immediately, as defined in the study protocol. There were no fatalities during the procedure or during the 10 days of follow-up.

The mean weight of the animals was $25.5 \mathrm{~kg}$ before and $24 \mathrm{~kg}$ after the POP. During follow-up, no sign of suffering or a change in behaviour was noted.

\section{Gastric emptying}

The mean duration of gastric emptying $(T 1 / 2)$ in the four pigs before POP was 188.47 minutes $( \pm 87.03 \mathrm{SD}$ ). After POP, the $T 1 / 2$ was 2.2-fold shorter, with an average duration of 84.5 minutes $( \pm 35.74 \mathrm{SD})$. This difference was statistically significant $(P=$ 0.029). The duration of gastric emptying in each of the four pigs before and after POP is shown in 0 Fig. 2 .

\section{Histology}

Necropsy confirmed the only perforation that developed during endoscopy as a pneumoperitoneum without peritonitis.

In agreement with the endoscopic observations, sectioning of the pyloric muscle in each pig was histologically complete $>90 \%$ of the length and thickness of the muscle). An example of pathologic assessment of the pylorus is shown in $\bullet$ Fig. 3 .

\section{Discussion \\ $\nabla$}

Peroral endoscopic pyloromyotomy is a new functional technique that evolved from endoscopic submucosal dissection (ESD) and POEM. In Europe, the anaesthetised pig is considered to be a valid training model and recommended for ESD learning. Our study demonstrates that the same model can be adapted for POP learning and development. The endoscopists who participated in this study had no previous experience with either POP or POEM, but each had already conducted 60 ESDs in animal models and 30 ESDs in human patients. Thus, even among these inexperienced operators, the feasibility of POP was $100 \%$. The duration of the procedure was typically 55 minutes. Because the anatomy and histology of pigs and humans are similar, this porcine model not only allows training in this technique but also familiarises physicians with the muscular fibrous section of the pylorus. The success of this learning approach was demonstrated by the ability of operators with experience only in ESD to identify the muscular fibres easily during each procedure. This was confirmed by

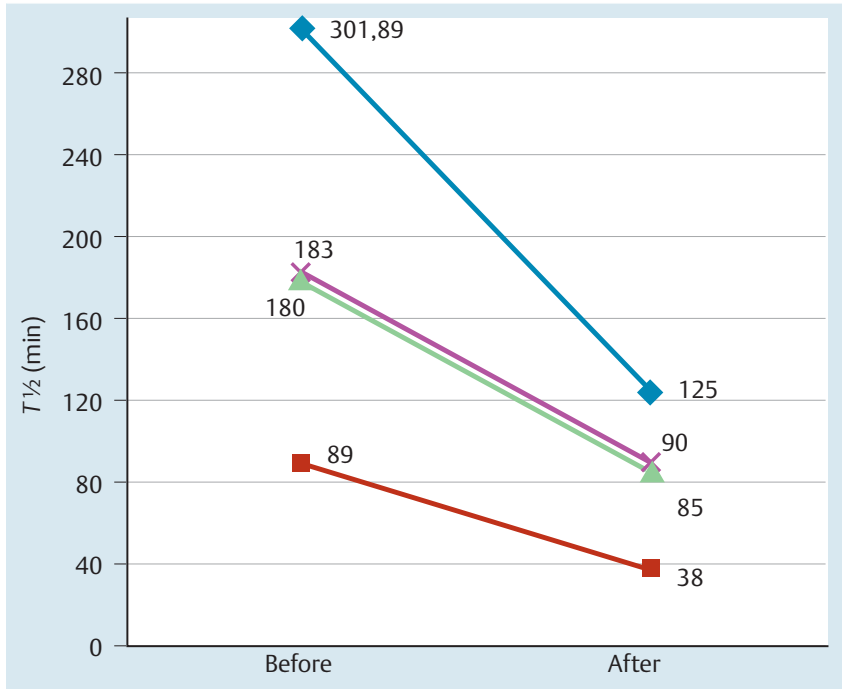

Fig.2 Individual results for gastric emptying scintigraphy showing $T \frac{1}{2}$ values before and after peroral endoscopic pylorotomy (POP).

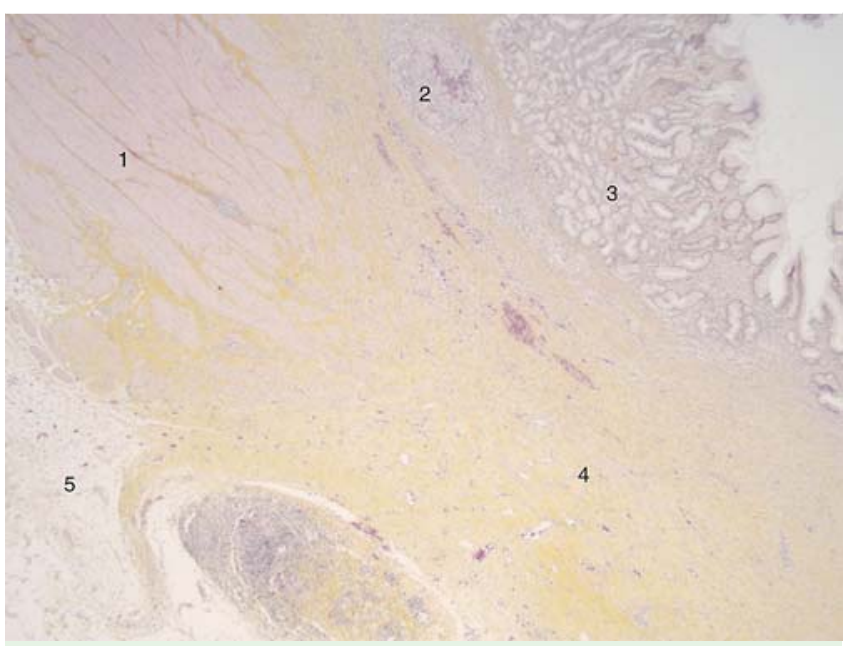

Fig. 3 Pathologic assessment of a complete section of the pylorus (horizontal axis and haematoxylin and eosin (H\&E) staining): 1 , normal gastric muscularis propria; 2 , granuloma replacing the submucosa (tunnel dissection); 3, normal gastric mucosa; 4, fibrosis replacing the pyloric muscle (area of the pyloromyotomy); 5 , serosa and subserosa. 
histologic analysis, which verified the completeness of the myotomy in all four pigs.

These encouraging results on animals recommend this model for surgeons who plan to operate on humans. In fact, endoscopy is more difficult in pigs than in humans because of differences in their gastric anatomy. The porcine stomach is U-shaped, resulting in higher pressure on the gastroscope, and it has larger gastric loops. Seen in retrovision, the pylorus is positioned next to the cardia, which renders POP more difficult. The J-shaped stomach of humans should facilitate the procedure.

The safety of POP was indicated by the occurrence of a perforation in only one pig, which was uncomplicated. The perforation occurred during endoscopy and was inconsequential because of the establishment of a submucosal dissection tunnel, which allowed the perforation site to be isolated from the gastric secretions. However, this complication was due to the many gastric loops in the U-shaped porcine stomach. In humans, the systematic use of $\mathrm{CO}_{2}$ insufflation and the J-shape of the stomach would minimise the risk of perforation during the procedure. There is currently no animal model validated for the evaluation of endoscopic or surgical procedures aimed to treat gastroparesis. The simplicity of our study protocol, combined with the availability of a gastric emptying study, highlight the feasibility of using mini-pigs as an animal model. Our study therefore provides a proof of concept of the beneficial effect of POP on gastric emptying, even in healthy pigs. Moreover, it is the first study to use scintigraphy for thorough evaluation of the procedure.

In 2011, in the first animal study on POP, Kawai et al. [12] used manometry to assess its effects on pyloric pressure, but they were unable to evaluate the impact on gastric emptying. We determined that the $T \frac{1}{2}$ of gastric emptying in healthy pigs was accelerated more than twofold after POP, confirming the efficiency of the procedure.

The variation of gastric emptying duration between each pig before and after POP could be compared with healthy humans. Indeed a wide inter-individual variation has been described in pivotal studies. But, as it is the first study using gastric emptying scintigraphy in pigs, there is no reference concerning these data [13].

To better characterise and understand the mechanism underlying the efficacy of POP, we are currently working with a porcine model of gastroparesis after surgical vagotomy. By examining gastric emptying under these conditions, we will be able to assess the efficiency not only of POP but also of other endoscopic/surgical procedures.

Pylorospasm is a common finding in patients with gastroparesis [7]; thus, POP may be the treatment of choice for refractory gastroparesis.

POP could also represent an alternative procedure in hypertrophic pyloric stenosis if suppliers are able to miniaturize their devices for use in infants (operative channel of paediatric gastroscopes: $2 \mathrm{~mm}$ ).

\section{Conclusion}

$\nabla$

A twofold decrease in $T 1 / 2$ of gastric emptying was achieved after POP, as verified by gastric emptying scintigraphy. The efficacy of the procedure provides indirect proof of the involvement of the pyloric ring in delayed gastric emptying and suggests new therapies for patients with gastroparesis.

Our protocol combining gastric emptying scintigraphy and POP validated the use of anaesthetised mini-pigs as a learning and training model for POP or other endoscopic/surgical procedures related to gastric emptying. Pathologic confirmation of pyloric sectioning, the low complication rate, and the absence of mortality demonstrate the feasibility and safety of POP, even when performed by physicians without experience in POEM.

A prospective study on gastroparetic pigs after surgical vagotomy is ongoing in parallel with prospective work on patients with refractory gastroparesis and a significantly impaired quality of life.

\section{Competing interests: None}

\section{References}

1 Camilleri M, Parkman HP, Shafi MA et al. American College of Gastroenterology. Clinical guideline: management of gastroparesis. Am J Gastroenterol 2013; 108: 18-37; quiz 38

2 Jung H-K, Choung RS, Locke GR et al. The incidence, prevalence, and outcomes of patients with gastroparesis in Olmsted County, Minnesota, from 1996 to 2006. Gastroenterology 2009; 136: 1225-1233

3 de la Loge C, Trudeau E, Marquis P et al. Cross-cultural development and validation of a patient self-administered questionnaire to assess quality of life in upper gastrointestinal disorders: the PAGI-QOL. Qual Life Res 2004; 13: 1751-1762

4 Szarka LA, Camilleri M. Gastric emptying. Clin Gastroenterol Hepatol 2009; 7: 823-827

5 Hasler WL. Gastroparesis. Curr Opin Gastroenterol 2012; 28: 621 -628

6 Nguyen LA, Snape WJ. Clinical presentation and pathophysiology of gastroparesis. Gastroenterol Clin North Am 2015; 44: 21 - 30

7 Gourcerol G, Ducrotté P. Editorial: impaired fasting pyloric compliance in gastroparesis and the benefits of therapeutic pyloric dilatation authors' reply. Aliment Pharmacol Ther 2015; 41: 908

8 Sarosiek I, Davis B, Eichler E et al. Surgical approaches to treatment of gastroparesis: gastric electrical stimulation, pyloroplasty, total gastrectomy and enteral feeding tubes. Gastroenterol Clin North Am 2015; 44: $151-167$

9 Youn YH, Minami H, Chiu PWY et al. Peroral endoscopic myotomy for treating achalasia and esophageal motility disorders. J Neurogastroenterol Motil 2016; 22: 14-24

10 Khashab MA, Stein E, Clarke JO et al. Gastric peroral endoscopic myotomy for refractory gastroparesis: first human endoscopic pyloromyotomy (with video). Gastrointest Endosc 2013; 78: 764-768

11 Shlomovitz E, Pescarus R, Cassera MA et al. Early human experience with per-oral endoscopic pyloromyotomy (POP). Surg Endosc 2015; 29: $543-551$

12 Kawai M, Peretta S, Burckhardt $O$ et al. Endoscopic pyloromyotomy: a new concept of minimally invasive surgery for pyloric stenosis. Endoscopy 2012; 44: $169-173$

13 Tougas G, Eaker EY, Abell TL et al. Assessment of gastric emptying using a low fat meal: establishment of international control values. Am J Gastroenterol 2000; 95: 1456-1462 